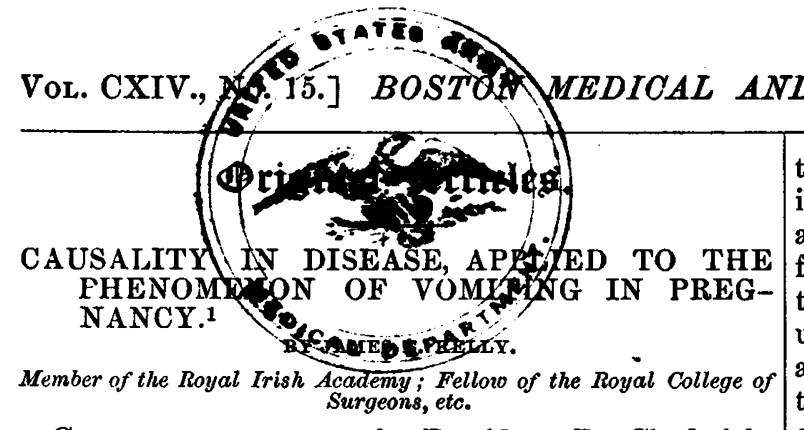

Genteemen, your worthy President, Dr. Chadwick, has aroused me from an intellectual lethargy of more than two ycars, by asking me to elaborate my impromptu remarks relative to the advantages to be derived from the application of physiological principles to the elucidation of one of the most obscure and intractible conditions of disease, namely, vomiting in pregnancy. As a man who has devoted some years of his professional career to physiological teaching and who, in consequence of the custom prevalent in the higher ranks of the medical profession in Great Britain and Ireland, of transferring all obstetrical cases to the care of specialists who limit their practice to that field, was not interested in the phenomenon, except in a casual manner, I consented, perhaps without a due appreciation of the extent and importance of the undertaking. I had but commenced to arrange my ideas, when I discovered that this apparently limited subject was, in reality, one of the most interesting and profound which I have encountered in the domain of biology, embracing the great questions of life, nutrition, cell-proliferation, nervous action, and a host of other subjects for the considerations of which, a few weeks were but as a single moment. I have been additionally hampered by the feeling that my production is but an addendum to the practical paper which was read to you by Dr. Doe, and also, owing to the knowledge that I stand between the Society and a communication which my slight acquaintance with Dr. Cushing convinces me is one of great interest and practical value. While writing this paper I have fully realized the significance of the saying of the celebrated Pascal, when apologising for the length of one of his compositions he said, "I had not time to write it shorter." Any one busily engaged in practice will sympathize with me in my difficulty and condone many of my omissions. It may be said with great apparent justice that I have submitted to this section some ideas which would be more suitable to a biological society, but as no such body exists in Boston, I have no alternative; also, that $I$ have not discriminated with sufficient care between my personal ideas on physiological and other questions and those which are more generally accepted, but, I must confess, I have treated the matter freely and hurriedly, and $I$ give it to you as it came to me, rough-hewn and unpolished.

The form of vomiting which particularly engages our attention to-night is that which takes place during pregnancy. It constitutes one terminal of a causal chain which has its primary link in the gravid uterus or its vicinity, where the energy which produces the phenomenon is developed. In order to limit our field of thought I shall review the causes of vomiting generally, and attempt to classify them. Firstly, vomiting is induced by irritation applied directly to the stomach: $a$, by the effects of irritants introduced into the organ; $b$, by modification of structure as we find in inflammation, simple ulcer, and in carcinoma; $c$, by

1 Read before the Suffolk District Medical Society, Section of Obstetrics and Gynæcology, Jan. 20, 1886.

the disturbance of function occurring in dyspepsia and in the vicarious secretions which take place in uræmia and lithiasis, diseases, by the way, to which the pregnant female is peculiarly liable; $d$, by the mechanical irritation resulting from the encroachment of the gravid uterus and other abdominal tumors. In this class the afferent impressions are conveyed by the pneumogastric nerves to the presiding centres, from whence the efferent impressions are dispatched to the involved muscles. Secondly, vomiting also results from impressions originating in other organs, or from the introduction of certain agents into the circulation, which disturb the control of the vomiting centre in the medulla oblongata, by exciting it to over action, or by diminishing its inhibitory power. These disturbing influences, except when of toxic origin, are grouped under the common term, "reflex action," and the severity of the symptoms depends upon, $a$, the intensity and duration of the disturbing energy; $b$, the susceptibility of the centre to its effects, and $c$, the responsivity and capacity of the involved muscular structures. In this class, vomiting may be due, $a$, to pain or shock; $b$, to those strange "nerve-storms" which originate in some emotional cerebral disturbance; $c$, to excessive action or nutrition in any organ, as in the gravid uterus, and $d$, to irritation, the term being used in a very comprehensive sense, applied to nerve-endings, nerve fibres, or nerve centres, in direct or indirect continuity with the vomiting centre. In many cases of vomiting more than one of these causes contribute to the result, and the recognition of their co-existence must have an important influence upon the success of the treatment.

Vomiting is a complex operation resulting from the complemental action of the muscular coats of the stomach and of the muscles of respiration. These two factors are usually combined, although it is possible for on of them to accomplish the act alone, as is seen in the so-called "cerebral vomiting" and in the wellknown experiment of Magendie upon the dog. In that interesting symptom, "cerebral vomiting," the patient, owing to a grave cerebral lesion, is lying unconscious, without any apparent movement other than simply gaping or slowly opening his mouth, he discharges the contents of his stomach. This evacuation is produced by the peristaltic action of the walls of the stomach. In the experiment upon the dog, the stomach is removed and in its place is introduced a bladder containing fluid. When the necessary stimulus is applied, the muscles of respiration contract, the bladder is compressed against the diaphragm by the recti and the broad muscles of the abdomen, and its contents are expelled by their simultaneous contraction. Thus, vomiting, except when caused by the unaided action of the stomach, in common with sighing, yawning, coughing, hiccoughing and retching, is a disturbance of the rythmical or alternate order of the acts of inspiration and expiration. This fact, in connection with the proximity of the presiding nerve centres in the medulla oblongata, should not be forgotten in the consideration of the etiology and the therapeutics of the phenomenon in pregnancy. In that distressing modification, retching, it is probable that owing to the absence of the preparatory dilatation of the cardiac orifice, the force of the respiratory muscles is fruitlessly expended upon the stomach. I am satisfied that the observation of the relative preponderance of the two factors in a case of vomiting would conduce to its 
comprehension and to its more efficient treatment. Owing to the comparatively free communication between the uterus and the respiratory muscles, we should anticipate a preponderance of the influence of the respiratory muscles in reflex vomiting and of the muscular walls of the stomach in the irritative form.

In consequence of the interlacement of the numerous branches of the sympathetic and the cerebro-spinal nerves, and of the presence of many ganglia in the abdomen, we find it difficult to resolve the connections between the uterus on one side, and the stomach and the respiratory organs on the other; but we can explain the apparent complexity by dividing them into two classes; first, the nervous communications between the uterus and ovary, where the stimulus may sometimes originate, and the stomach; second, those which connect the same organs with the respiratory muscles. The hypogastric plexus which supplies the pelvic viscera with its nerves is, necessarily, a common link in all the routes. In the first class there are two routes, one, through the sympathetic nerves from the hypogastric plexus to the solar plexus and thence, by its gastric subdivision, to the stomach; the second, from the hypogastric plexus through its spinal roots to the spinal cord and thus to the medulla oblongata, where, in the vomiting centre the afferent impressions are converted into an efferent influence, which is transmitted to the stomach through the pneumogastric nerves. In the second class, by one route we can trace the connection from the hypogastric plexus, by its spinal roots to the spinal cord through which it may be transmitted directly to the respiratory muscles by the intercostal, phrenic and other nerves, and by the second, having reached the cord by the same route, the nervous energy is conveyed to the vomiting centre whence it is transmitted through the cord to the respiratory muscles by the same nerves as in the ast instance. The second route in each class is that which is the more familiar. In the diagram shown I have indicated the courses by the use of different colors.

Before proceeding further I shall recall a few general principles, which, if time permitted, I should endeavor to apply to our present subject.

Energy is a general term applied to all forces, such as heat, chemical action, light, sound, electricity, gravitation, etc., which have the power of modifying matter by overcoming resistance, and under certain circumstances, and in the presence of certain forms of matter are convertible each into all or any of the others.

Kinetic energy is the result of molecular or molar change, produced by the action of another force, which disturbs the condition of rest or stability, termed equilibrium.

A special energy requires for its manifestation a special modification of matter.

Vital energy is only manifested in the presence of protoplasm, and it is the product of the two factors, life and protoplasm.

All functional and constructive vital energies, are the result of molecular change, which disturbs the equilibrium of the energy associated with protoplasm.

Vital energy manifests itself under various modifications, as nutrition, vital heat, nervous energy, muscular action, etc., and it is correlative with the various physical energies.

All vital energy as well as organic matter must be traced from its source to its destination before the enigma of Life is solved.

Owing to the specialization of function, nutrition and reproduction, the essential energies in the lower conditions of life, are, in the anima kingdom, subdivided into various forms which are reciprocally productive of one another.

Nutrition is the proximate result of vital energy, and all vital phenomena are its consequences.

All forms of vital energy, but especially nervous energy, are, like light, modified by diffusion, intensification, dispersion, reflection, interception, and conversion.

According to a principle analogous to that of the correlation of forces, it is probable that similar influences govern the vital and the physical energies, a speculation which our observation corroborates in many instances.

Death, in the organic world, is the condition of absolute equilibrium of the vital energies, associated with the suspension of nutrition.

Health is the condition in which the sum of the vital energies is limited to certain "normal" bounds, and the various sub-energies bear to each other certain "normal" proportions. They are in equilibrium.

Disease is that condition which results from the transgression of these limits, in either direction, by the sum of the vital energy, or by one or more of its forms ; also by the disturbance of the relative proportions existing, within those limits, between the several manifestations of vital energy.

Local disease is an approximate term, indicating a comparatively limited disturbance, which has only local manifestations.

Owing to the great length of this paper and to its suggestive nature, 1 have endeavored to combine many of the physiological considerations with the therapeutics of the subject, a method which prevents me from doing justice to my topic or to myself. The therapeutical indications have the same basis as in all other applications of "rational" treatment. We must endeavor, first, to modify the disturbance of structure or function which originated the abnormal state of energy in the uterus; second, to transform or otherwise modify, the resulting nervous energy; third, to divert or obstruct it in the channel which conducts it to the abnormally acting structures, either in the efferent nerves which convey it to the nerve-centres, in the nerve-centres themselves, or in the efferent nerves leading to the structures; fourth, to nullify its influence upon these structures, namely, the coats of the stomach, and the muscles of respiration. In the selection of the method of treatment, the practitioner is guided by his knowledge, his judgment and his conclusions.

I shall now speak of the greater frequency of this grave condition in the primiparæ, and I should attempt to explain it by one or more of the following considerations: First, displacements of the uterus are more under observation and control in the multiparæ; second, owing to previous pregnancies, the ligaments of the uterus and its walls, if not modified by inflammation, are in a more favorable condition for the essential changes ; third, in the primiparæ, the adaptability to maternity, and the power of resisting its ills, are tested, with the result of the elimination of many of the aspiring candidates for that high honor ; fourth. it is possible that the susceptibility of the various nervous 
centres may become blunted by the test to which they have been submitted in previous pregnancies, or by the still greater strain of parturition.

Almost invariably, nervous energy in the female is in a comparatively unstable condition, and during gestation it is still more liable to violent disturbances, as we often witness in eclampsia, mania, hysteria, and many other neuroses. 'This is easily understood when we recall the immense amount of energy convertible into the nervous form, which must be developed in the uterus during the construction, within a comparatively limited period, of a machine combining a greater complexity of structure and a greater variety of function than the collective wisdom of the human mind can conceive. We also observe, in pregnant females, an exaltation of the functions generally, as we should expect in conjunction with the adoption of the responsibility and the support of another individual.

We can have little conception of the influences which effect a nervous impression from its origin until it rtaches its destination, if it should pass through one or more ganglia. The central nervous system is a combination of ganglia; a ganglion contains many multipolar cells, which form interminable communications with one another, and with innumerable nerve-cells and fibres external to the ganglion. An impression arriving at the simplest ganglion is liable to have its power diminished to a homœopathic degree by subdivision, or, by combining with other nervous currents which reach the ganglion from other sources, it may proceed on its course, intensified a thousand-fold. Here, it would be permissible to speculate upon the influence which induces the current to adopt a special avenue of exit, for on it is based Education, in its highest and in its lowest relations. Its application to our present theme is shown by the remarks of your worthy President and others, who spoke of administering small and frequent quantities of food, of the chewing of "sprucegum," "pop-corn," and other incentitives to frequent deglutition. This would indicate the training of the nerve-centres, by repeated impressions, to adopt a special form of nervous action, resulting in a suspension of the perverted condition which produced the emesis. Another application of the principle of education is afforded us by the observations of some of the other speakers, who mentioned cases in which vomiting contiuued after abortion had been produced. Assuming the correctness of the diagnosis of the cause of the persistency, I am at a loss to explain the occurrence, except as the result of "habit," which is synonymous with the modification of the structure of the presiding centre, resulting, most probably, from the increase of the conductivity or the size of the nerve-fibres conveying the efferent impressions to the stomach. This speculation is based upon the general principles that increase of function, cateris paribus, is associated with increase of structure, and that energy travels in the direction of the least resistance. As we do not recognize the existence of effect without cause, this is a subject which I should gladly develop if a suitable opportunity should offer itself. While we remember the educational advantage derivable from increased salivation, we must not forget that the saliva, being an alkaline fluid, may have a sedative action upon the irritated mucous membrane. Although we can hardly expect any of this influence to be due to the small quantity of Sulphocyanide of Potassium, it is interesting to remember its presence in the saliva, and perhaps, to ask our- selves, if it be in the power of the biologist to teach us how to increase its secretion, a speculation which may not be fruitless in other fields. As a gentleman alluded to the frequent coincidence of salivation with vomiting, I wish to recall the close relation of the salivary and the vomiting centres in the medulla oblongata, which association may afford a clue to many other complex phenomena. Your President also mentioned some cases in which the patients derived great advantage from the habit of taking nourishment immediately before composing themselves to sleep, a valuable suggestion, which is sustained by two important considerations. One is the fact that in over-action of the nervous centres, food in the stomach, by exciting the process of digestion, and converting the nervous into the vito-chemical energy, acts as a sedative; and the other is based upon the well-known principle, which is hardly contined to medical subjects, that weakness and irritability are closely associated, and consequently, any measure having a tendency to relieve the former should ameliorate the latter.

Although we cannot expect to interfere, to any great extent, with the normal progress of gestation, the consideration of a few of its most salient points will be of interest, and perhaps of advantage. In the development of the uterine walls, we must consider two factors of especial importance to this question. One is the rapid cell-proliferation which takes place in all the tissues; the other is the modification of the form of the cavity. I shall again remind you of the immense volume of energy which is generated by cellnutrition and reproduction. They also produce tension upon the nerve-endings and the fibres, if any disproportion should exist between the expansion and the proliferation. If we were to attempt any interference with the natural process of uterine development, we should look to those remedies which are useful in counteracting excessive development in disease. Bloodletting stands at the head of the list, which is very limited, containing only Lead, Mercury, Potassium, and a few minor drugs. Ipecacuanha may enhance its high standing by proving itself useful in this conjunction. One other medicine I should wish to mention, which appears to possess a marked influence upon uterine action. I speak of Viburnum Prunifolium, and I should be curious to learn if its sedative effects were of any benefit in uterine vomiting. 'The union of the cervical with the general cavity of the uterus, and their remarkable expansion subsequently, can only occur at the expense of much traction and modelling pressure. In the fact that the uterus resembles the other hollow organs in the special development of the nerves which guard its outlet, we may have a clue to the vomiting and to the beneficial results of dilatation of the os uteri, a procedure which is held in high estimation. Its action is two-fold: first, forcible and sudden removal of the tension which irritated the nerves and their endings ; and second, the application of the wellknown principle of " nerve stretching" to the involved fibres. I may here remark that an apparatus which would apply instantaneous and general dilatation to the orifice, should be more efficacious than that method which one of the gentleman described at our last meeting. He divaricated the blades of a forceps which he introduced within the os, one and a half inches in one direction, and then he repeated the manœuvre, transversely to the same extent. It is hardly necessary to say that the second step of the operation did not in- 
crease the size of the os uteri, any more than it would effect any other circular aperture, which would yield to an equal extent in every direction.

If we cannot prevent the development of the uterine energy, we may cast about for some method of diverting it from the hypogastric plexus, and here we are reminded of the treatment which is directed to the cervix and to the os uteri. We occasionally hear of benefit being derived from the application of Iodine and Nitrate of Silver, and I should attempt to explain their action by quoting a principle from that prince among men, the late James Syme, of Edinburgh: "Pain, hæmorrhage, inflammation, increased nutrition, and excited secretion, take the place of one another, so that they may be regarded as equivalents of action." Following up this clue, and recalling the theory of nervous energy, a wider field for well-founded experiment would develop itself. By the application of the actual or the potential cautery, we should expect to produce more marked and enduring results. The former is much facilitated by that manageable little instrument, Paquelin's cautery, while Nitric Acid, Potassa Fusa, and the Ethylate of Sodium, afford ready methods of applying the latter. Acupuncture, by its efficacy, induces us to employ it in many cases, although we can hardly hazard a speculation on its mode of action. In whatever manner it modifies energy, which it appears to do by producing a sedative influence, it is possible that its application to the cervix of the gravid uterus may be associated with some benefit. Another expedient may produce a similar influence in some cases of vomiting, as in other forms of violent nervous perturbation. I allude to ovarian pressure, which is so valuable in hystero-epilepsy. It may be applied by a truss or other apparatus externally, or by a combination of external and vaginal pressure.

Assuming that we cannot modify the uterine energy, we may consider if we can intercept it in its passage to the nerve centres, or induce it to adopt another goal. We find that the resources of therapeutics are limited in the number of the paralyzers and depressants of the afferent nerve fibres, but when we recognize among them such potent medicine as Aconite, Physostigma, and Nitrite of Amyl, we cannot allow this indication to pass unnoticed. As to the application of the principle of counter irritation, we should endeavor to discover if any portions of the integment have special connection - "linked spots" - with the uterus or the involved centres, at which this expedient may be more effectively applied either in the form of Corrigan's button, or as an issue, rather than as a simple blister.

Regarding secretory energy as a correlative of the other vital energies, we should expect to find it as a frequent and obvious result of the uterine influences, as in salivation and excessive secretion of the gastric juice or mucus. It would be interesting to observe if we could utilize this suggestion by diverting the surplus energy to the elaboration of some secretions which would promote its diffusion. This line of thought naturally reminds us of the secretory action of the skin, an old and well-established avenue for the elimination of heat and other forms of energy. In Pilocarpine we have a drug of remarkable efficacy, and we possess many other medicines which may combine this influence with other advantages. Another basis for this suggestion is the proximity of the presiding sweat centre, to the vomiting, respiratory, and other important centres in the medulla oblongata. In this conjunction it is convenient to consider the influence exerted by the morbid displacements and the normal elevation of the uterus. We must recall for an instant the observation of gentlemen of ability and opportunity, such as Graily Hewitt, who noted that the majority of the displacements, associated with vomiting, were antiversions. This is as we should expect when we remember that the nervous supply of the uterus reaches it from below and behind, and therefore, in this form of displacement, the fibres would be most liable to straining, tension and irritation. Most probably in cases of retroversion the irritation applied to the nerve fibres would be by pressure between the uterus and the pelvis, or some other viscus, such as an impacted rectum. For such cases the conditions would suggest the rational treatment, by replacement or by the evacuation of the distended viscera. A very interesting question arises in those cases which result from the abnormal resistance to the essential elevation of the uterus. It is possible that benefit may be derived from the application of some power within the vagina which with a justifiable amount of force and rapidity, would elevate the uterus en masse, and thus liberate the nerves from the resisting ligaments, and at the same time exert upon them the sedative influence of nerve stretching. A suitable apparatus might be devised, by modifying that instrument known as the "cup pessary" and adding to it a bandle. Here I am reminded that the application of heat and cold according to the multiple modes known to the "craft," by bath, bag, or douche, may be beneficial. It is possible that heat in the form of a bath may diffuse, direct or conduct from the body the energy otherwise concentrated on the stomach. This may be a partial explanation of its well-established sedative influence. In massage we have ar1other mode of producing a somewhat similar effect. By the stimulation which it applies to all the structures of the body as well as by its manifest influence upon the general nutrition, it may diffuse the superabundant vital energy. This is a remedy which admits of many applications. The utility of cold in many forms of disease needs no commendation; but the cold bath or a cold douche may exert two influences worthy of being specially canvassed. One is by the removal of heat and the consequent conversion of the nervous energy to supply the place of the abstracted calorific energy; again the well-known influence which cold exerts over development may be utilized. I have not alluded to the effect produced upon the arterioles because I am inclined to consider that our pathologists have been running that one intermediate function to death to the disadvantage of many investigations which appear to possess much interest and importance.

When the nervous current reaches the great centres, such as the spinal cord and medulla oblongata, according to the present knowledge which we possess, we must concentrate our forces upon attempting to destroy their conductivity and their power of reflecting the nervous impressions. Whether some philosopher shall ever discover the manner in which the various drugs act upon the nervous centres I cannot forecast, but I am satisfied that any exhaustive mind, which applies itself to this subject cannot be content with the explanation which the biologists offer us. According to our light we may divide the drugs from which we may expect aid into the depressors and paralyzers of the medulla and the spinal cord. Among those medicines 
from which we would anticipate the most potent influence upon the vomiting centre in the medulla, are Varatrum Viride, Aconite, the Chlorals, Nitrite of Amyl, and a host of others ; while amoug those which exert the most distinct influence on the cord, in addition to some of the last mentioned, are Physostigma, Hyoscyamia, Antimony, and Conium. By the action of those drugs the nervous centres are deprived of the power of transmitting to the engaged muscles the nervous impressions which they receive from the uterus, and, according as we deem the cord or the centre in the medulla the more active in the development of vomiting, so should we select the more appropriate remedy. If the former should be the case, Physostigma is the most promising agent, and if the latter, Veratrum Viride or its principle, Veratria. I may mention that upon physiological principles the much used and abused remedy, "Atropia and morphia," administered hypodermically should be more closely studied, as the former tetanizes the cord, increases reflex action and stimulates the respiratory centre, while the morphia to a lesser extent produces similar effects in some of its manifestations. Nux Vomica may be admissable in some cases owing to its acting as a stimulator upon all parts of the spinal cord by which effect it may diffuse the energy which otherwise would be concentrated on the centres of vomiting and respiration. I shall allude to another medicine here which may be found useful owing to the remarkable sedative effect it manifests in dyspnœea, an influence which may extend from that of respiration to the adjacent vomiting centre, in the floor of the fourth ventricle; I speak of Quebracho.

The muscles of respiration afford us the next field for combatting the malign influence developed in the uterus and transmitted to those powerful agents. This object can be effected in one of two ways, either by paralyzing the muscles or by producing a condition which will prevent the compression of the stomach. In Curara, that powerful drug of such obscure origin, so potent in hydrophobia and in tetanus, and which is used by vivisectionists, when desirous of producing absolute immobility without interfering with the other functions of the nervous system, we find an agent imperatively demanding a full and fair trial in a disease which frequently submits to us nothing better than the selection of the lesser of two evils, the death of the mother or the destruction of the offspring. The other expedient, by which the stomach is protected from the pressure of the respiratory muscles, is tracheotomy, by which the fixation of the diaphragm is rendered impossible, and consequently the contraction of the abdominal muscles is almost nullified, the stomach being evacuated only by the action of its own walls.

Finally if the dread alternative, abortion, ever should be considered necessary, it would be well if it were performed with gentleness, safety, and certainty. It would appear more difficult to destroy the ovum than one who has a purely theoretical knowledge of the subject could imagine, when we have one experienced gentleman mentioning a case in which all his efforts were ineffectual, and another, equally accomplished, telling of having discovered that he had passed Simpson's sound ten inches in a direction contrary to that of the uterus, which was antiflexed. It occurs to me that Electricity either in the form of the interrupted or the continuous current, as the indications would suggest the expulsion or the destruction of the foetus, ap- plied by means of a supra-pubic and an intra-uterine electrode, would have enabled the gentlemen to complete this unsavory operation without any additionally disagreeable contretemps.

In conclusion I would ask my audience to remember the object of this paper. It is to indicate to what extent physiology is capable of adding to the resources of the practitioner, by giving him a comprehensive view of obscure diseases, and enabling him rationally to select the most suitable among the recognized remedies, and to bring to the alleviation of human misery other resources of which he had not previously thought in conjunction with the particular condition upon which he is desirous of concentrating his knowledge and his intellect. To borrow the expression of a distinguished Irish philosopher, I would indicate how, by " reasoning alone, which merely unfolds as it were, and develops, what was, in a manner, wrapped up and implied in our previous knowledge, but which we are often as unaware of, to all practical purposes, till brought before us, as if it had been wholly beyond our reach," we may increase the efficiency and the dignity of medicine. I have given rein to speculation and transgressed the crudely practical limits of the question, because $I$ wished to maintain the suggestive nature of the paper. Were I dealing with the subject from the responsible standpoint of the practitioner, I should have weighed every word, and investigated the efficacy and the safety of every new application of every expedient which I have suggested, but in the primary stages of "truth seeking" we are allowed a greater liberty. I shall have attained my object if $I$ induce some of my audience to apply that unerring touchstone, thought the real philosopher's stone, which turns all things to gold - to the question before us, and I shall be gratified still more if my views are combated, for " Truth like a torch, the more it's shook it shines."

\section{SOME OF THE LESS COMMON FORMS OF MASTOID DISEASE.}

BY J. ORNE GREEN, M.D.

MAstord osteitis, as it has been well called by Politzer, because we may get any of the conditions which accompany inflammation of bone, assumes a variety of external appearances and symptoms most of which are well understood and readily diagnosticated. The most common form is due to extension of the inflammation which exists within the mastoid cells, through the external cortex to the outside of the bone, with resulting external periostitis and subperiosteal abscess with or without a carious fistula through the bone. This expresses itself by tenderness of the bone on pressure, odema of the soft structures and finally fluctuation. In these cases the external appearances odema, swelling and fluctuation are found on the flat external surface of the mastoid process and an incision parallel with and near the insertion of the auricle when carried through the periosteum will evacuate the pus, if the case has gone on to full suppuration, or, if it has not yet proceeded so far, a slight raising of the periosteum to one side or the other will usually expose the spot through which the inflammation has come from the inside, a small spot of the bone being inflamed, or softened by decalcification, or perhaps distinctly carious. The point to which I would direct attention in 